Rabaska

Revue d'ethnologie de l'Amérique française

\title{
Centre canadien de recherche sur les francophonies en milieu minoritaire (Université de Régina)
}

\section{Frédéric Dupré}

Volume 3, 2005

URI : https://id.erudit.org/iderudit/201754ar

DOI : https://doi.org/10.7202/201754ar

Aller au sommaire du numéro

Éditeur(s)

Société québécoise d'ethnologie

ISSN

1703-7433 (imprimé)

1916-7350 (numérique)

Découvrir la revue

Citer ce document

Dupré, F. (2005). Centre canadien de recherche sur les francophonies en milieu minoritaire (Université de Régina). Rabaska, 3, 224-225.

https://doi.org/10.7202/201754ar d'utilisation que vous pouvez consulter en ligne. 


\section{Centre canadien de recherche}

\section{sur les francophonies en milieu minoritaire}

Institut français

Université de Regina

3737 Wascana Parkway

Regina (Saskatchewan) S4S 0A2
Téléphone : (306) 337-2357

Télécopieur : (306) 585-5183

Courriel : frederic.dupre@uregina.ca

Le Centre canadien de recherche sur les francophonies en milieu minoritaire (CRFM) élabore, de façon multidisciplinaire et inter-institutionnelle, un programme de recherche portant en priorité sur l'expérience de la communauté fransaskoise tout en s'ouvrant à celle d'autres francophonies canadiennes et internationales qui cherchent à s'affirmer en milieu minoritaire. Le Centre favorise aussi des recherches permettant de comparer cette expérience francophone à celle d'autres peuples et groupes ethnoculturels qui se trouvent en milieu minoritaire, tels les Métis et les Premières Nations du Canada. Dans ce sens, le CRFM collabore étroitement avec la communauté fransaskoise pour favoriser la création de projets de recherche qui répondent à ses préoccupations actuelles.

Fondé en janvier 2005, le CRFM est un centre jeune, dynamique et innovateur. Le professeur Peter Dorrington en est actuellement le directeur et Frédéric Dupré, le coordonnateur. Le CRFM vient, ce printemps, de lancer son premier concours de subventions de recherche qui vise à appuyer la recherche sur les communautés francophones en milieu minoritaire et plus particulièrement en Saskatchewan. Une particularité originale de ce concours est que, entre autres, il offre un programme de subvention à l'intention des chercheurs communautaires. Cette initiative vise à encourager la collaboration scientifique avec le milieu communautaire ainsi qu'à renforcer les capacités de recherche au sein même des communautés. Le CRFM a annoncé à la fin juillet le financement d'une dizaine de projets de recherche dans le cadre de ce concours.

Malgré son jeune âge, le Centre se positionne comme un acteur de premier plan dans la recherche sur les francophones. Il est déjà très actif dans le domaine de la recherche grâce à diverses collaborations à des projets ayant trait à des aspects historiques et contemporains de problématique touchant particulièrement les francophonies canadiennes en milieu minoritaire. Le CRFM a encouragé concrètement la création d'une équipe de chercheurs ancrée dans l'Ouest pour étudier à l'échelle nationale le lien entre la culture, la 
langue et l'accès au système des soins de santé en milieu minoritaire. La lettre d'intention déposée aux Instituts de recherche en santé du Canada (IRSC) a été acceptée et l'équipe prépare en ce moment la demande définitive. C'est là une initiative qui s'est développée en partenariat avec le Consortium national de formation en santé (CNFs) et des chercheurs de plusieurs universités.

Le CRFM est également un partenaire de recherche au sein du projet international (France, Canada) «Inventaire des lieux de mémoire de la Nouvelle-France ». Au sein de cette équipe, le Centre s'occupe du recensement et de l'inscription des lieux de mémoire de la Nouvelle-France situés dans l'Ouest canadien au sein de la banque de données accessible sur la toile.

Le CRFM s'engage également à mener une réflexion sur les relations entre les francophones et d'autres communautés en situation minoritaire au Canada. Dans ce sens, du 20 au 23 octobre 2005, le Centre organise un colloque international intitulé « Résistances et convergences : stratégies identitaires des francophones et des Métis de l'Ouest canadien ». Ce colloque constitue un événement historique dans la mesure où il rassemblera pour la première fois les communautés francophone et métisse pour entamer une réflexion collective sur leurs stratégies de renforcement communautaire et d'épanouissement identitaire. Le colloque offrira quarante-six communications de scientifiques de renom, des soirées culturelles où artistes métis et francophones partageront la même scène et deux tables rondes communautaires qui permettront d'entamer un dialogue constructif entre ces deux communautés apparentées.

Le CRFM compte s'imposer comme un chef de file de la recherche qui s'articule à la jonction de l'universitaire, du communautaire et du minoritaire. Tout en privilégiant la recherche auprès de la communauté fransaskoise, le Centre développe un programme de recherche novateur qui s'ouvre aux autres francophonies en milieu minoritaire et qui favorise une perspective interculturelle. 